S sciendo

\title{
Is Rationality Normative?
}

John Broome

Corpus Christi College, University of Oxford

Disputatio Vol. 2, No. 23

November 2007

DOI: $10.2478 /$ disp-2007-0008

ISSN: 0873-626X 


\title{
Is rationality normative?
}

\author{
John Broome \\ Corpus Christi College, University of Oxford
}

\begin{abstract}
Rationality requires various things of you. For example, it requires you not to have contradictory beliefs, and to intend what you believe is a necessary means to an end that you intend. Suppose rationality requires you to F. Does this fact constitute a reason for you to F? Does it even follow from this fact that you have a reason to F? I examine these questions and reach a sceptical conclusion about them. I can find no satisfactory argument to show that either has the answer 'yes'. I consider the idea that rationality is normative for instrumental reasons, because it helps you to achieve some of the things you ought to achieve. I also consider the idea that rationality consists in responding correctly to reasons. I reject both.
\end{abstract}

\section{The normative question}

Rationality requires various things of you. For example, it requires you not to have contradictory beliefs, to believe what follows by modus ponens from things you believe, to intend what you believe to be a necessary means to an end that you intend, and to intend to do what you believe you ought to do. These are only rough formulations, and rough formulations are perhaps enough for this paper.

However, for the sake of accuracy, I shall give more formal statements of the requirement that you believe what follows by modus ponens from things you believe, and the requirement that you intend to do what you believe you ought to do. These ones will come up later in the paper. They are:

Modus ponens. Rationality requires of $N$ that, if $N$ believes $p$ and $N$ believes that if $p$ then $q$, and if it matters to $N$ whether $q$, then $N$ believes $q$.

Krasia. Rationality requires of $N$ that, if $N$ believes that she herself ought to $F$, and if $N$ believes that she herself will $F$ if and only if she herself intends to $F$, then $N$ intends to $F$.

Disputatio, Vol. II, No. 23, November 2007 (special issue) 
These are technically requirement-schemata; individual requirements are obtained by making appropriate substitutions for the schematic letters: the name of a person for ' $N$ ', a sentence for ' $p$ ' and a verb phrase for ' $F$ '. Each formula states a requirement on $N$ 's contemporaneous mental states. To make that explicit, I could have added 'at $t$ ' to each of the attitudes that are mentioned in the formulae; I did not do so simply to make the formulae less complicated. In the formula for Krasia, 'she herself' is a compound reflexive pronoun; it represents in indirect speech the first-person pronoun 'I' in direct speech. ${ }^{1}$ The formulae contain some clauses that do not appear in the informal statements; I leave you to work out why they are needed.

I shall use less formal language in this paper. Even so, I shall sometimes use a schematic letter to stand for a verb phrase. For instance, I might say 'rationality requires you to $F$.' This is unattractive, but sadly English does not provide a good alternative. My ' $F$ ' may stand for a complex phrase, such as 'intend what you believe to be a means to an end that you intend.' There is no generic verb in English with a broad enough meaning to stand in for all the substitutions that may be needed. 'Do' is too narrow; it covers little more than actions. However, because a schematic letter is not always tolerable, in places I shall artificially adopt 'achieve' as my generic verb. This is not accurate English, but fortunately the contexts make it possible. I hope you will accept it now that I have warned you.

This paper considers whether rationality is normative. It is automatically normative in one sense. Rationality is a system of requirements or rules. It therefore sets up a notion of correctness: following the rules is correct according to the rules. That by itself makes it normative in one sense, because in one sense 'normative' simply means to do with norms, rules or correctness. Any source of requirements is normative in this sense. For example, Catholicism is. Catholicism requires you to abstain from meat on Fridays. This is a rule, and it is incorrect according to Catholicism to eat meat on Fridays. So Catholicism is normative in this sense.

But I do not use 'normative' in that sense. In my sense, it means to do with ought or reasons. Given a rule or a requirement, we can ask whether you ought to follow it, or whether you have a reason to do so. Is there any reason for you to abstain from meat on Fridays, for

\footnotetext{
${ }^{1}$ See Castañeda 1968.
} 
instance? I shall ask the corresponding question about the requirements of rationality. 'Requirement' is not a normative word in my sense. $^{2}$ When I say rationality requires this or that of you, I do not mean anything normative in my sense. Nevertheless, it seems plausible that rationality actually is normative. Is it? I shall call this the 'normative question' about rationality. This paper looks at various possible arguments for the answer 'yes', and rejects them all.

What does the normative question mean, more exactly? The claim that rationality is normative comes in various strengths. The strongest is:

Strong normativity. Necessarily, if rationality requires $N$ to $F$, then $N$ ought to $F$ because rationality requires $N$ to $F$.

There is another way to say this. When you ought to $F$ because $X$, we say that $X$ is a reason for you to $F$. When, necessarily, you ought to $F$ if $X$, we say that $X$ is sufficient for it to be the case that you ought to $F$. When the two things are true together, we may say that $X$ is $a$ sufficient reason for you to $F .^{3}$ So strong normativity says that, if rationality requires you to $F$, that fact is a sufficient reason for you to $F$.

This claim is implicit in the way many philosophers write, including me in the past. It is common to describe requirements of rationality using the normative term 'ought'. For example, saying 'You ought not to have contradictory beliefs' is a common way of expressing the requirement not to have contradictory beliefs. In adopting this style of expression, I was implicitly assuming strong normativity. ${ }^{4}$

On the other hand, some philosophers think rationality has a normative force that is defeasible. They think that, if on some occasion your having contradictory beliefs would be very beneficial - perhaps it would prevent a war - then it is not the case that you ought not to have contradictory beliefs. Nevertheless, these philosophers think that rationality requires you not to have contradictory beliefs, and they think this is a consideration that counts against your having them. So they think the rational requirement not to have contradictory beliefs

\footnotetext{
${ }^{2}$ I have examined the meaning of 'requirement' in Broome 2007b.

${ }^{3}$ Some authors use the term 'sufficient reason' differently. By 'a sufficient reason to $F^{\prime}$ they mean a reason that is sufficient to make it permissible for you to $F$. I mean a reason that is sufficient to make it the case that you ought to $F$.

${ }^{4}$ Andrew Reisner persuaded me to change my practice.
} 
is a pro tanto reason for not having them. They claim that rationality is normative in a second, weaker way:

Medium normativity. Necessarily, if rationality requires $N$ to $F$, that fact is a reason for $N$ to $F$.

I mean 'a reason' in this formula to include both sufficient reasons and pro tanto reasons. So medium normativity is entailed by strong normativity, but it is weaker than strong normativity.

If you believe medium normativity but not strong normativity, you will think that the reasons generated by rationality sometimes conflict with reasons that issue from other sources of normativity, such as morality or prudence. But you will probably believe that conflicts of this sort are rare. Rationality is principally concerned with coherence among your attitudes such as your beliefs and intentions, whereas morality, prudence and other sources of normativity are rarely concerned with those things. Rationality has a domain of application where it is pretty much on its own. Examples of conflict between rationality and other sources of requirements tend to be farfetched, like my example of a preventing a war. So according to medium normativity, when rationality requires something of you, it will normally be the case that you ought to achieve that thing.

A yet weaker view is:

Weak normativity. Necessarily, if rationality requires $N$ to $F$, there is a reason for $N$ to $F$.

According to weak normativity, that rationality requires you to $F$ entails that you have a reason - either sufficient or pro tanto - to $F$, but the fact that rationality requires you to $F$ may not itself be the reason. Many philosophers think rationality consists in responding correctly to reasons - a view I shall discuss in section 3. They may well accept weak normativity but neither of the stronger views. They do not think the fact that rationality requires something of you is itself a reason to achieve that thing. Still, because they think rationality consists in responding correctly to reasons, they think rationality would not require something of you unless there was a reason for you to achieve that thing.

Weak normativity is not really a version of the claim that rationality is normative. It associates rationality with normativity, but it does 
not claim that rationality is a source of normativity. Nevertheless, in this paper I shall concentrate principally on weak normativity. That is because I shall reject even the arguments for that weak claim. Strong and medium normativity both entail weak normativity, so rejecting weak normativity entails rejecting both of them. They are both genuine versions of the claim that rationality is normative.

I do not disbelieve weak normativity. Indeed, I am inclined to believe even medium normativity. An important paper by Niko Kolodny (2005) has a stronger conclusion than mine. It concludes that rationality is actually not normative. But I am not convinced by Kolodny's arguments, for reasons I cannot go into here. ${ }^{5}$ I am agnostic about this question.

Compare the corresponding normative question for morality. Medium normativity is surely true of morality; it is surely true that, necessarily, if morality requires you to $F$, that fact is a reason for you to $F$. But it is notoriously difficult to find a convincing explanation of why. Rationality may be in the same position. It may be that rationality is indeed normative, but it is hard to explain why. The fact that I cannot do so may be just because I am not clever enough.

\section{Rationality and reasons}

Many philosophers unhesitatingly assume there is some conceptual connection between rationality and reasons. ${ }^{6}$ More specifically, they assume that acting contrary to reasons is irrational. ${ }^{7}$ Why make this assumption? There are certainly connections between the words 'rational' and 'reason.' For one thing, they have the same Latin root. But, although etymology can be suggestive, it gives no real ground for thinking there is a connection between the concepts.

More confusingly, the word 'reason' has various senses, and in one of them it refers to the faculty of reason. 'Reason' used this way is a mass noun - a noun that has no plural. Our faculty of rationality (which I shall mention again in section 4) is plainly part of, or perhaps

\footnotetext{
${ }^{5}$ See Broome 2007a.

${ }^{6}$ Just one example is Smith 2004.

${ }^{7}$ Again just one example: Williams 1981.
} 
all of, our faculty of reason. So there is certainly a conceptual connection between rationality and reason in this sense.

But the word 'reason' that appears in my formulae for medium and weak normativity does not have that sense. It is a count noun a noun that has a plural. Its plural is 'reasons'. Just because rationality is conceptually connected with the faculty of reason, it does not follow that it is conceptually connected with reasons.

When David Hume said

'Tis not contrary to reason to prefer the destruction of the whole world to the scratching of my finger. ${ }^{8}$

he was using 'reason' to refer to the faculty of reason. Since rationality is a part of this faculty, Hume's remark implies that it would not be irrational to have this preference. (Hume himself does not use the word 'irrational'.) However, it does not imply that there is no reason not to have it. We should not attribute that extraordinary view to Hume.

At this point, Hume is saying nothing about reasons. In the Treatise, he rarely uses the count noun 'reason' in a normative sense, and never in the section entitled 'Of the influencing motives of the will' that contains this remark about preference. It would obviously be very immoral to prefer the destruction of the whole world to the scratching of your finger, and therefore you ought not to have this preference. Since Hume had no less of a moral sense than the rest of us, we may fairly assume he would have agreed you ought not to have it. The preference is contrary to morality, he might have said. But since Hume did not think morality arises from the faculty of reason, it was perfectly consistent for him to say that the preference is not contrary to reason, this faculty.

Had Hume been willing to speak of normative reasons, I assume he would have agreed that morality gives you a reason, in the normative sense, not to have this preference. He would have agreed the preference is contrary to this reason, that is. Some philosophers have been more shocked by Hume's remark than they need have been. ${ }^{9}$

\footnotetext{
${ }^{8}$ Hume 1978, book 2, part 3, section 3.

${ }^{9}$ Johnston (1989: 161) is an example.
} 


\section{Responding correctly to reasons}

The confusion of words could explain why many philosophers associate reasons and rationality. But is there really any conceptual connection that gives the answer 'yes' to the normative question about rationality? It is very commonly said that rationality consists in responding correctly to reasons. If this were so, it might be the connection I have just asked for. At least, it might support weak normativity.

It would not infallibly do so, because the idea that rationality consists in responding correctly to reasons has two alternative readings. On a narrow-scope reading it means, first, that whenever there is a reason for you to $F$ then rationality requires you to respond correctly to this reason and, second, that this is all rationality requires of you. On a wide-scope reading it means, first, that rationality requires of you that whenever there is a reason for you to $F$ you respond correctly to this reason and, second, that this is all rationality requires of you. The wide-scope reading does not support weak normativity, but the narrow-scope reading does. Given the narrow-scope reading, if rationality requires you to $F$, that can only be because there is a reason for you to $F$. Weak normativity follows.

Does rationality consist in responding correctly to reasons, under either reading? There is a quick objection to the idea that it does. On some occasion, there might be a reason for you to achieve something but, without any irrationality on your part, you might not believe this reason exists. If you do not believe it exists, then you might well not respond correctly to it, and your failure will not imply any failure of your rationality. Therefore, rationality cannot consist in responding correctly to reasons.

For example, suppose the fish in front of you contains salmonella. This is a reason for you not to eat it. But there may be no obvious evidence that it contains salmonella. So you might not believe it contains salmonella, and you might eat it, and nevertheless you might be rational. So you are rational even though you do not respond correctly to the reason.

Many philosophers find this quick objection convincing. As a result, few support precisely the idea that rationality consists in responding correctly to reasons. Instead, they support a modified idea that connects rationality with the reasons you believe to exist rather than with actual reasons. They say that rationality consists in responding correctly to the reasons that you believe to exist, or something of that sort. 
I know no quick objection to this idea. But it does not support weak normativity. The narrow-scope reading of this view goes as follows: first, whenever you believe there is a reason for you to $F$ then rationality requires you to respond correctly to this believed reason and, second, this is all rationality requires of you. We can derive the conclusion that if rationality requires you to $F$, that can only be because you believe there is a reason for you to $F$. But weak normativity does not follow, because you might believe there is a reason when actually there is not. Mistakes about reasons are possible.

So if the quick objection to the original idea is sound, we can get no support for weak normativity from any conceptual connection that holds between rationality and responding correctly to reasons. Is the quick objection sound? I know of one response that may be made to it. The objection depends on two assumptions. One is that there can be a reason for you to achieve something that, without any irrationality, you might not believe exists. The second is that, if you do not believe a particular reason exists, you may fail to respond correctly to this reason, without any irrationality on your part. On the face of it, both these assumptions seem hard to doubt. But one theory of rationality circumvents the quick objection, and casts doubt on them.

I shall call it 'the theory of attitudinal reasons,' for a reason that will soon appear. It assumes that all the requirements of rationality are in one respect like the examples of requirements I mentioned at the beginning of this paper. In my examples, rationality requires particular relations to hold among your attitudes. It requires you to have or not have a particular belief if you have other particular beliefs; it requires you to have a particular intention if you have other particular intentions and beliefs; and so on. The theory supposes that all rational requirements have this character. According to the theory of attitudinal reasons, then, your rationality consists in the holding of appropriate relations among your attitudes. That is its first feature.

Its next feature is that it understands the requirements of rationality in a particular way. It takes each requirement to imply that some of your attitudes constitute reasons for your having other attitudes. Take as an example the requirement modus ponens set out in section 1. One instance of it is that rationality requires of you that, if you believe it is raining and you believe that if it is raining the snow will melt, you believe the snow will melt. (For the sake of setting out the theory of attitudinal reasons, I shall ignore the condition that it matters to you whether the snow will melt.) Suppose you believe it is 
raining and you believe that if it is raining the snow will melt. Let us call these beliefs your 'premise attitudes.' Let us call the belief that the snow will melt the 'conclusion attitude.' According to the theory, the premise attitudes together constitute a reason to have the conclusion attitude. Moreover, according to the theory, this is a sufficient reason: it makes it the case that you ought to have the conclusion attitude. Furthermore, according to the theory, to respond correctly to this reason is to have the conclusion attitude.

We are taking it for granted that rationality requires you to have the conclusion attitude if you have the premise attitudes. Therefore, you are irrational if you have the premise attitudes but do not have the conclusion attitude. As the theory of attitudinal reasons understands the requirement, you are irrational if you do not respond correctly to the reason in this case. So the theory implies that rationality consists in responding correctly to reasons. The reasons are of a particular type; they are constituted by the premise attitudes that are specified in requirements of rationality. The theory could allow there to be reasons of other types as well. Those ones would have to be unconnected with rationality. But for simplicity I shall assume that reasons of this type are the only ones it recognizes.

How does the theory of attitudinal reasons circumvent the quick objection? It must deny one or other of the assumptions that this objection relies on. It could deny the first. It could say that, when you have a reason to have some conclusion attitude, you must be irrational if you do not believe the reason exists. This is not entirely implausible. The reason consists of your premise attitudes, and it is not entirely implausible that, if you have attitudes of this sort, you are irrational unless you believe you have them.

However, I think the theory would do better to deny the second assumption. It would do better to say that, even if you do not believe the reason exists, nevertheless you are irrational if you do not respond to it correctly. This is plausible, given that the reason is constituted by your premise attitudes. Plausibly, you can come to have the conclusion attitude through a process of reasoning that does not require you to believe you have the premise attitudes. That indeed is my own view: I think that reasoning does not require you to have 
second-order beliefs about your attitudes. ${ }^{10}$ You can reason directly from the contents of your premise attitudes. In the example, you can reason from the contents of your premise beliefs - the proposition that it is raining and the proposition that if it is raining the snow will melt — to a new belief that the snow will melt. This reasoning process does not require you to have any beliefs about your attitudes; you do not need to believe you believe it is raining, for instance. If this is right, then you can respond correctly to your reason — the premise attitudes — without believing this reason exists. Furthermore, plausibly, you are irrational if you do not do so.

The upshot is that the theory of attitudinal reasons could offer a defence of the idea that rationality consists in responding correctly to reasons. It offers to defend only a very restricted version of the idea: that rationality consists in responding correctly to reasons that are constituted out of premise states. But still, this is some defence.

However, the defence is not yet complete. There is first of all a matter of scope to attend to. Weak normativity says that, when rationality requires of you that you $F$, there is a reason for you to $F$. In the cases we are considering, what rationality requires of you is that, if you have the premise attitudes, you have the conclusion attitude. So we must take $F$ to be the conditional that, if you have the premise attitudes, you have the conclusion attitude. But the theory of attitudinal reasons does not claim you have a reason to satisfy this conditional. It claims that, if you have the premise attitudes, you have a reason to have the conclusion attitude. So it does not support weak normativity directly. However, I doubt this is a serious difficulty. I expect it would be easy to argue that a reason to have the conclusion attitude is also a reason to satisfy the conditional $F$. At any rate, I shall ignore this difficulty.

The serious problem lies elsewhere. The theory of attitudinal reasons simply assumes that your premise attitudes constitute a reason to have the conclusion attitude. But this is to beg the normative question. We all agree that rationality requires of you that, if you have the premise attitudes, you have the conclusion attitude. This is not a normative claim, since I do not use 'requires' as a normative word. But the theory of attitudinal reasons understands this requirement in a

\footnotetext{
${ }^{10}$ My objection to what I call the 'second-order model' of reasoning is briefly set out in Broome 2006.
} 
normative way. It takes it to imply that the premise attitudes constitute a reason for having the conclusion attitude. It expresses the rational requirement using the normative term 'a reason.' But it has no right to do that until it has established that the rational requirement does indeed make a normative connection between the premise attitudes and the conclusion attitude. Whether this is so is just the normative question.

The theory of attitudinal reasons is not truly a defence of the idea that rationality consists in responding correctly to reasons. It is simply an assertion that it is so, within a limited domain. It offers no argument for the claim that rationality is normative.

\section{An instrumental reason to be rational}

Section 3 shows that we can get no support for weak normativity from the idea that rationality consists in responding correctly to reasons. I am now going to pursue a different approach to the normative question. I shall pursue it in a generous spirit; I shall do my best to make it work. On its behalf, I shall make assumptions I cannot properly justify, and rely on arguments that are frankly rough. I have already said that my ultimate conclusion will be sceptical: I know no good grounds for thinking rationality is normative. Given that, a generous spirit is appropriate. I want to see how far it can take us in finding grounds.

The approach I am going to pursue starts by recognizing that, if rationality is indeed normative, that seems likely to be because of what we can achieve by being rational. It seems likely to be for instrumental reasons, as I shall put it.

Since some philosophers give a different meaning from mine to the word 'instrumental,' I need to be clear about mine. I am not suggesting the requirements of rationality might be normative because satisfying them is a way of satisfying our desires. I am suggesting they might be normative because satisfying them is a way of achieving some of the things we ought to achieve. I ignore the possibility of total scepticism about normativity; I take it for granted there are some things we ought to do, some things we ought to hope for, some things we ought to believe, some things we ought not to do, not to hope for, not to believe, and so on. There are some $F$ s such that we ought to $F$. Rationality seems plausibly a good means of coming to $F$ in many instances when 
we ought to $F$ - of achieving much of what we ought to achieve. Perhaps this explains why we have a reason to satisfy rational requirements. The rest of this paper explores that idea.

It cannot be applied directly to individual requirements. If there is a reason for you to satisfy each individual requirement, the reason cannot always be a directly instrumental one. That is because satisfying a particular requirement will sometimes not contribute to your achieving anything you ought to achieve. Indeed, it will sometimes prevent you from achieving something you ought to achieve. For example, suppose you believe you ought not to sell your car, but your belief is false and actually you ought to sell it. If you satisfy the requirement krasia of rationality on this occasion, you will intend not to sell your car. As a result of your intention, you will probably not sell it. But you ought to sell it. So in this case, satisfying a rational requirement will probably prevent you from doing something you ought to do.

Often no doubt, when rationality requires you to achieve something, you do have a direct instrumental reason to achieve it. But that is not necessarily so. There is therefore no direct instrumental argument for weak normativity.

Is there an indirect argument? We can start by noticing it is much more plausible that, for directly instrumental reasons, you ought to have the rational faculty. By 'the rational faculty' I mean the bundle of dispositions and abilities that causes you to satisfy many of rational requirements. This is only a rough definition because of the vague term 'many,' but it will serve for my purposes. The connection between the rational faculty and satisfying individual rational requirements is causal.

Possessing the rational faculty is plausibly part of the best means of achieving much of what you ought to achieve. By 'best' I mean better than other means that are psychologically possible for you. In principle, there might be an alternative faculty that could form part of an even better means of achieving much of what you ought to achieve. On some occasions, the rational faculty will steer you wrong; the car examples illustrates how it will sometimes cause you to fail to achieve something you ought to achieve. The alternative faculty would be like the rational faculty, but altered a little to correct some of those glitches. But the necessary alterations would depend on complex circumstances in the outside world, and I assume this alternative would not be psychologically possible for you. 
I shall therefore assume that the rational faculty is part of the best means of achieving much of what you ought to achieve. Given that, two claims are plausible. First, you ought to have the rational faculty. Second, if you ought to have the rational faculty, that is because having it is part of the best means of achieving much of what you ought to achieve.

I am sorry to say I cannot offer a proper argument to support the first of these claims. One difficulty standing in the way is that a proper argument would have to depend on an accurate account of the way in which normativity is transmitted from ends to means. If you ought to achieve some end, how does that determine what means you ought to take to that end? In our particular case, I am assuming the rational faculty is part of the best means to the end of achieving things you ought to achieve. How does it follow that you ought to have the rational faculty? An accurate answer to this question would be complicated. It is not clear that you ought always to take the best means to an end that you intend. ${ }^{11}$ The best means may be bad in some way that is unconnected with the end. If so, perhaps you ought to take some means other than the best one.

To deal with this difficulty properly would take all the resources of decision theory - decision theory deals with the relation between ends and means, and it can be interpreted in normative terms. But there is no need for that sort of detail in this paper. I am working in a generous spirit, and looking only for rough arguments. Let us accept the plausible claim that you ought to have the rational faculty.

The second claim is that, if the first claim is true, it is true because having the rational faculty is part of the best means of achieving much of what you ought to achieve. I can give this claim some support by describing a world where the rational faculty is not instrumentally effective in this way.

I assume it is only a contingent fact, if it is a fact at all, that the rational faculty is part of the best means of achieving much of what you ought to achieve. I assume there could be quirky worlds where that is not so. In a quirky world, people with the rational faculty generally satisfy the same requirements of rationality as rational people do in our world. They generally intend to do the things they believe they ought to do; they generally do not have contradictory beliefs; they

\footnotetext{
${ }^{11}$ As Carsten Nielsen helpfully reminded me.
} 
generally believe what follows by modus ponens from things they believe; and so on. But because of the way causal processes work in their world, satisfying the requirements of rationality tends to be unsuccessful. These people tend not to end up having the beliefs they ought to have, doing the things they ought to do, and so on. They do not achieve much of what they ought to achieve.

In a quirky world there are also people who do not have the rational faculty. Those people do just what they feel like doing, believe whatever comes into their heads, and so on. The causal processes in their world bring it about that these people achieve much of what they ought to achieve.

Plausibly, it is not the case that people in a quirky world ought to have the rational faculty, since it is not a means of achieving much of what they ought to achieve. This suggests that, if the rational faculty were not instrumentally successful, it would not be the case that we ought to have it. So it supports the claim that, if we ought to have the rational faculty, that is because it is instrumentally successful.

On the basis of these rough arguments, let us now suppose that you ought to have the rational faculty, and that this is so for instrumental reasons. Could it be because of this that you ought to satisfy the individual requirements of rationality? If so, it would give us a good answer to the normative question. Not only would you have a reason to satisfy rational requirements, but the reason would have to be that rationality requires it. Medium normativity would be true. Rationality would be a genuine source of normativity.

By contrast, if there had been a direct instrumental argument for satisfying rational requirements, it would have established only weak normativity. The reason for satisfying each requirement would have been what could be achieved by doing so. If we are to show on instrumental grounds that rationality is normative, we therefore need the indirect argument we are now investigating, rather than a direct one.

But the indirect argument does make it necessary to add one qualification. ${ }^{12}$ If rationality is normative on instrumental grounds, it will not be normative in worlds where the rational faculty is not instrumentally successful. It will not be normative in quirky worlds, therefore. This means that medium normativity as it is stated in section 1 will not be true. It will need to be weakened to something like:

${ }^{12}$ Alexander Bird and Hannes Leitgeb pointed this out to me. 
necessarily except in quirky worlds, if rationality requires $N$ to $F$, that fact is a reason for $N$ to $F$.

\section{Still no answer to the normative question}

Sadly, this is all pie in the sky. So far as I can tell, the indirect instrumental argument does not go through. We are assuming you ought to have the rational faculty, and that this is so for instrumental reasons. It does not follow by any means of inference I can find that rationality is normative.

If you have the rational faculty, it will cause you to satisfy many individual requirements of rationality. But it does not follow by any general principle of inference that there is a reason for you to satisfy those requirements. 'You ought to $F$; if you $F$, your Fing will cause you to $G$; so there is a reason for you to $G$ ' is not a valid pattern of inference.

This should be uncontroversial. Suppose you ought to take some drug to cure your serious disease. Suppose the drug has the side effect of causing you to feel unsteady on your feet. It does not follow that you have a reason to feel unsteady on your feet. To be sure, if you take the drug and as a result feel unsteady on your feet, we might say there is a reason why you feel unsteady on your feet. That would simply be another way of saying there is an explanation of why you feel unsteady on your feet. 'The reason why' in this context has a non-normative meaning equivalent to 'the explanation of why' this is yet another sense of the promiscuous word 'reason.' There is no suggestion that there is a reason, in any normative sense, for you to feel unsteady on your feet. In general, normativity is not transmitted from something to a causal effect of that thing.

Still, given the premise that you ought to have the rational faculty, perhaps we might nevertheless be able to derive the conclusion that rationality is normative. The very general inference pattern I set out is invalid, but there might be some more specific way to put through the derivation. For example, perhaps we might be able to develop a valid argument using some extra premise. I can only report that I have found no way to do so.

Would a different, parallel argument be more successful $?^{13}$ I defined your rational faculty to be a cause of your satisfying individual

\footnotetext{
${ }^{13}$ Philip Pettit made this suggestion to me.
} 
requirements of rationality. I could have based the argument on some different property of yours, which has a necessary, non-causal connection with satisfying the individual requirements. For example, let us say you are fully rational if and only if you satisfy all the requirements of rationality. Being fully rational and satisfying any particular requirement of rationality are then logically connected. Logically necessarily, if you are fully rational, you satisfy each particular requirement. Could this give us the basis of an argument?

Suppose we could demonstrate that you ought to be fully rational. Then we would be able to call on a different pattern of inference to derive the conclusion that you have a reason to satisfy a particular requirement. We could use the pattern: 'You ought to $F$; logically necessarily, if you $F$, you $G$; so there is a reason for you to $G$ '. We could substitute 'be fully rational' for ' $F$ ' and 'satisfy a particular rational requirement' for ' $G$ '. This would give us a different argument for the normativity of rationality.

But I see two difficulties with this parallel argument. First, the new inference pattern is questionable. Suppose you ought to buy a can of pain and decorate your kitchen. It follows by this pattern of inference that you ought to buy a can of paint. But suppose you are not going to decorate your kitchen; you have no intention of doing so, and you will not do it. Then it seems obvious that there may be no reason for you to buy a can of paint. If you are not going to decorate your kitchen, it may be entirely pointless to buy one. This example suggests the inference pattern is invalid.

However, that pattern is nevertheless defensible. Standard deontic logic even validates the stronger pattern: 'You ought to $F$; logically necessarily, if you $F$, you $G$; so you ought to $G$ '. So for safety I shall not put much weight on this difficulty.

The other difficulty is more severe. I can see no satisfactory way of arguing for the premise that you ought to be fully rational. The argument is supposed to be an instrumental one, so it would depend on showing that being fully rational is instrumentally effective. How could that be shown?

To make the corresponding argument that you ought to have the rational faculty, I assumed that having this faculty is part of the best means of achieving much of what you ought to achieve. By 'best' I meant best among those means that are psychologically possible for you. Granted that assumption, it is plausible that you ought to have the rational faculty. 
But being fully rational is not even psychologically possible for you; no one could be as rational as that. And once we are looking into psychologically impossible properties, being fully rational would not be the most instrumentally effective among them. To be fully rational is to satisfy each of the individual requirements of rationality. But the example of selling your car shows it is more instrumentally effective for you to fail to satisfy a few of these requirements. By doing so, you will achieve more of what you ought to achieve. Given all this, I do not see how one could argue that you ought to have the psychologically impossible property of being fully rational.

In sum, I have found no successful argument on instrumental grounds for the conclusion that rationality is normative. Neither the rational faculty nor the property of being fully rational seems to offer a sound basis for an argument. It still seems intuitively plausible to me that rationality is normative. Moreover, it seems plausible to me that this is so for a broadly instrumental reason. But I am sorry to say that I simply cannot find an argument.

\section{Conclusion}

After all this, I have been unable to show that the requirements of rationality are normative. I have failed to show even that weak normativity is true: that, necessarily, when rationality requires you to $F$, there is a reason for you to $F$. Often when rationality requires you to $F$, you have a reason to $F$, but I cannot guarantee this is necessarily so.

When we accuse someone of irrationality, we are surely criticizing her. How could we be entitled to do so if there is no reason for her to satisfy the requirements of rationality in the first place? Well, it may indeed be the cases that, necessarily, she has a reason to satisfy the requirements; I merely have not been able to show that is so. But even if she does not have a reason, there is another possible explanation of why a charge of irrationality is a criticism.

I said that, plausibly, you ought to have the rational faculty. This is for instrumental reasons. If you do not satisfy some particular rational requirement, that is evidence that you do not have this faculty, or at least that you do not have it to the highest degree. So it is evidence that you are failing to achieve something that, plausibly, you ought to achieve. That makes it grounds for justified criticism. If you fail to satisfy a particular rational requirement, that may show you have 
gone wrong. But where you have gone wrong may not be in failing to satisfy the requirement. You may have gone wrong in failing to have the rational faculty, which your failure is evidence of.

In any case, my conclusion is sceptical. I can find no grounds for thinking that rationality is normative. ${ }^{14}$

$$
\begin{array}{r}
\text { John Broome } \\
\text { Corpus Christi College } \\
\text { Oxford OX1 4JF, UK } \\
\text { john.broome@philosophy.ox.ac.uk }
\end{array}
$$

\section{References}

Broome, John. 2005. Does rationality give us reasons? Philosophical Issues 15: $321-37$.

Broome, John. 2006. 'Reasoning with preferences?' In Preference Formation and Well-Being, ed. by Serena Olsaretti. Cambridge: Cambridge University Press.

Broome, John. 2007a. Wide or narrow scope? Mind 116: 359-70.

Broome, John 2007b. 'Requirements'. In Homage à Wlodek: Philosophical Papers Dedicated to Wlodek Rabinowicz, ed. by Toni Rønnow-Rasmussen, Björn Petersson, Jonas Josefsson and Dan Egonsson, at www.fil.lu.se/HommageaWlodek/site/papper/BroomeJohn.pdf.

Castañeda, Hector-Neri. 1968. On the logic of attributions of selfknowledge to others. Journal of Philosophy 65: 439-56.

Hume, David. 1978. A Treatise of Human Nature. Edition by L. A. SelbyBigge and P. H. Nidditch. Oxford: Oxford University Press.

Johnston, Mark. 1989. Dispositional theories of value. Proceedings of the Aristotelian Society supp. vol. 63: 139-74.

Kolodny, Niko. 2005. Why be rational?. Mind 114: 509-63.

Smith, Michael. 2004. Humean rationality. In The Oxford Handbook of Rationality, ed. by Alfred R. Mele and Piers Rawling. Oxford: Oxford University Press.

Williams, Bernard. 1981. Internal and external reasons. In Moral Luck. Cambridge: Cambridge University Press.

${ }^{14}$ This paper has developed out of Broome 2005. It has benefited from the discussion at the European Congress of Analytical Philosophy, Lisbon 2005 - particularly from Philip Pettit's criticism — and from discussions at the many universities where I have subsequently presented it. 